\title{
Transtornos mentais comuns e associação com variáveis sociodemográficas e estressores ocupacionais: uma análise de gênero
}

\section{Common mental disorders and its association with sociodemographic variables and occupational stressors: a gender analysis}

\section{Trastornos mentales comunes y asociación con variables sociodemográficas y estresores ocupacionales: un análisis de género}

\section{Mary Sandra Carlotto*}

Pontifícia Universidade Católica do Rio Grande do Sul - PUC-RS, Porto Alegre, Rio Grande do Sul, Brasil

\section{Mariana Barcinski**}

Pontifícia Universidade Católica do Rio Grande do Sul - PUC-RS, Porto Alegre, Rio Grande do Sul, Brasil

\section{Rosália Fonseca***}

Tribunal de Justiça do Estado do Rio Grande do Sul, Porto Alegre, Rio Grande do Sul, Brasil

\begin{abstract}
RESUMO
Este estudo teve por objetivo identificar a prevalência de Transtornos Mentais Comuns (TMC) em servidores públicos da Justiça Estadual e verificar a existência de associações entre variáveis sociodemográficas e estressores ocupacionais entre homens e mulheres. A amostra aleatória constituiu-se de 541 servidores públicos, 354 mulheres e 187 homens, pertencentes a uma instituição do Poder Judiciário do Estado do Rio Grande do Sul, em 2011. Os instrumentos utilizados foram o Self Reporting Questionnaire (SRQ-20) para avaliar os Transtornos Mentais Comuns e um questionário composto por questões sobre dados sociodemográficos, laborais e estressores ocupacionais. Os resultados evidenciaram que as mulheres apresentam maior prevalência de TMC. Na relação entre TMC e variáveis sociodemográficas e estressores ocupacionais, de acordo com o sexo, verifica-se relação com o tempo de trabalho e com os estressores carga horária, diversidade, complexidade do trabalho e relação com chefia, colegas e ambiente social. Os dados foram discutidos a partir da compreensão sobre o mercado de trabalho contemporâneo e sua interface com as relações de gênero. Os resultados apontam a necessidade de intervenções diferenciadas nos grupos pesquisados.
\end{abstract}


Palavras-chave: transtornos mentais comuns, estresse ocupacional, gênero.

\begin{abstract}
This study aimed at investigating the prevalence of common mental disorders (CMD) in public servants of the State Court, as well as to verify the associations between sociodemographic variables and occupational stressors among men and women. The random sample was composed by 541 public servants, 354 women and 187 men, working in an institution of the Judiciary in Rio Grande do Sul, in 2011. The adopted instruments were the Self Reporting Questionnaire (SRQ-20), to evaluate common mental disorders and a questionnaire composed by items containing sociodemographic, work and occupational stressors data. Results showed that women present a higher prevalence of CMD. Regarding the relationship between sociodemographic variables and occupational stressors, according to sex, there is a relation with both working time and the following stressors: workload, work diversity, work complexity, and the relationship with bosses, colleagues, and social environment. The data were discussed from the understanding of contemporary work market, and its interface with gender relations. The results point to the need of distinct interventions with the researched groups.
\end{abstract}

Keywords: common mental disorders, occupational stress, gender.

\title{
RESUMEN
}

Este estudio tuvo por objetivo identificar la prevalencia de Trastornos Mentales Comunes (TMC) en funcionarios públicos de la Justicia Estadual y verificar la existencia de asociaciones entre variables sociodemográficas y estresores ocupacionales entre varones y mujeres. La muestra aleatoria se constituyó de 541 funcionarios públicos, 354 mujeres y 187 varones pertenecientes a una institución del poder judiciario del Estado del Rio Grande do Sul, en 2011. Los instrumentos utilizados fueron el Self Reporting Questionnaire (SRQ-20) para evaluar trastornos mentales comunes y un cuestionario compuesto por datos sociodemográficos, laborales y de estresores ocupacionales. Los resultados evidenciaron que las mujeres presentan mayor prevalencia de TMC. En la relación entre TMC y variables sociodemográficas y estresores ocupacionales, conforme el sexo, verificase relación con el tiempo de trabajo y con los estresores carga horaria, diversidad, complexidad del trabajo y relación con el jefe, compañeros y entorno social. Los datos fueron discutidos a partir de la comprensión del mercado de trabajo contemporáneo y su interface con las relaciones de género. Los resultados apuntan la necesidad de intervenciones diferenciadas en los grupos investigados.

Palabras-clave: trastornos mentales comunes, estrés ocupacional, género.

\section{Introdução}

Transtornos Mentais Comuns (TMC) são caracterizados como transtornos somatoformes, de ansiedade e de depressão (Mari \& Jorge, 2004). A sintomatologia constitui-se de insônia, fadiga, irritabilidade, esquecimento, dificuldade de concentração e queixas somáticas (Goldberg \& Huxley, 1992). Embora inicialmente não necessitem de tratamento psiquiátrico, causam grande impacto 
socioeconômico, principalmente pelos dias de trabalho perdidos e devido ao aumento da procura de seus portadores pelos serviços de saúde (Broadhead et al., 1990; Goldberg \& Huxley, 1992; Porto et al., 2006; Szeto \& Dobson, 2013).

De acordo com Lopes, Faerstein e Chor (2003), estudos realizados entre a população ocidental têm apontado uma variação de prevalência de $7 \%$ a $26 \%$ para os TMC. No Brasil, investigações revelam que sua prevalência tem se associado com o aumento da idade e é maior em mulheres, negros, pessoas separadas ou viúvas, com baixas escolaridade e renda (Lima et al., 1996; Ludermir \& Melo Filho, 2002). Os TMC podem estar associados às características demográficas, condições de vida, estrutura ocupacional (Ludermir \& Melo, 2002; Silva \& Meneses, 2008; Porto et al., 2006) e estressores ocupacionais (Araújo, Aquino, Menezes, Santos, \& Aguiar, 2003; Macedo et al., 2007).

Nos últimos anos, verifica-se uma tendência de intensificação do ritmo de trabalho, aumento de empregos altamente qualificados e 0 maior uso de informação e tecnologia de comunicação, que colocam exigências cada vez mais elevadas sobre as funções mentais dos trabalhadores (European Agency on Safety and Health at Work, 2007), com implicações importantes para sua saúde mental e ocorrência de transtornos relacionados a estressores ocupacionais (Nieuwenhuijsen, Bruinvels, \& Frings-Dresen, 2010).

O estresse ocupacional tem sido definido como a tensão decorrente do desequilibro entre a percepção das demandas de trabalho como um estressor e a avaliação por parte do trabalhador sobre sua capacidade em termos de conhecimentos e habilidades para manejálo adequadamente (Leka, Griffiths, \& Cox, 2003; Paschoal, \& Tamayo, 2004). O estresse ocupacional tem sido um dos grandes problemas do mundo moderno do trabalho, pois ocasiona diversos problemas de saúde (Divya, Girija, Tintu, \& Santhosh, 2013).

Trabalhadores em postos de trabalho de alto estresse tendem a ter maior prevalência de transtornos mentais (Szeto \& Dobson, 2013). Os efeitos do estresse ocupacional sobre a saúde do trabalhador, em um ambiente laboral em constante e intensa modificação, ainda, são pouco conhecidos e devem fazer parte da agenda de saúde pública (Macedo et al., 2007).

\subsection{Trabalho, adoecimento e gênero}

Os trabalhadores compartilham os perfis de adoecimento e morte da população em geral em função de sua idade, sexo, grupo social ou insercão em um grupo específico de risco. Neste sentido, o adoecimento deve ser entendido a partir da sua relação com variáveis sociodemográficas específicas e com aspectos característicos do 
processo de trabalho contemporâneo que implicam na ocorrência de estressores ocupacionais (Ministério da Saúde do Brasil, 2001).

Ao longo dos anos, verifica-se um expressivo aumento da participação das mulheres no mercado de trabalho, assim, tornandose necessário avaliar o impacto da variável sexo no adoecimento relacionado ao estresse laboral (Barnett, Marshall, Raudenbush, \& Brennan, 1993), principalmente porque alguns estudos têm evidenciado estresse mais elevado em mulheres (Areias \& Guimarães, 2004; Glise, Ahlborg, \& Jonsdottir, 2012; Theorell, Hammarström, Gustafsson, Hanson, \& Westerlund, 2014).

A categoria trabalho incorpora, historicamente, visíveis relações de desigualdade e de poder assimétrico entre homens e mulheres (Cyrino, 2009). Embora possam partilhar de condições de trabalho e de vida similares, há situações resultantes da própria organização social de gênero que estabelecem diferenças nos processos de enfermidade entre homens e mulheres. Portanto os problemas referentes ao trabalho enfrentados pelas mulheres não são os mesmos enfrentados pelo trabalhador do sexo masculino (Apple, 1995; Borsoi, 1995) e os riscos de adoecimento relacionados ao trabalho são diferentes para esses dois grupos (Areias \& Guimarães, 2004; Moreno, 1999).

Apesar de certa ruptura nas formas tradicionais de organização da vida social, que propicia significantes transformações nas condições das mulheres, ainda persistem relações hierárquicas de gênero, em que os homens assumem, na vida social e no trabalho, as posições dominantes e mais valorizadas (Diogo \& Coutinho, 2006; Cyrino, 2009).

Acerca da sobrecarga feminina, com a sobreposição das responsabilidades laborais e domésticas, Narvaz e Koller (2006) afirmam que, embora a família contemporânea esteja redefinindo os papéis masculinos e femininos no cuidado com a casa e com a prole, as mulheres ainda assumem grande parte das responsabilidades com as funções de reprodução. Mesmo quando elas não assumem diretamente a realização das tarefas com a casa, enfatiza a autora, cabe às mulheres a organização da vida doméstica através da delegação deste trabalho (usualmente a outra mulher) e da supervisão do mesmo.

Portanto, convivem na família contemporânea novas formas de organização, explicitadas pelo compartilhamento de tarefas domésticas e de cuidado, com arranjos tradicionais, segundo os quais o trabalho reprodutivo desempenhado no âmbito privado é entendido ainda como obrigação feminina, conquanto o compartilhamento das responsabilidades e das preocupações com a criação dos filhos e com as tarefas da casa já seja bastante comum (Apple, 1995; Borsoi, 1995). Heilborn (2004) ressalta o fato de que a ideologia igualitária, entre casais na atualidade, pressupõe uma simetria do ponto de vista 
do capital financeiro e cultural de homens e mulheres. Em outras palavras, uma relação simétrica, com o real compartilhamento das responsabilidades com a casa e com a prole, traduz-se como um ideal de relação típico de classes sociais mais favorecidas, em que a dependência financeira das mulheres não se coloca como uma realidade.

As relações entre os TMC e as desigualdades de gênero têm recebido destaque na literatura, uma vez que um dos resultados mais persistentes é que as mulheres apresentam uma prevalência duas a três vezes maior que a dos homens (Ludermir \& Lewis, 2005; Ludermir, 2008). Esse resultado vem se confirmando em diversos estudos (Carlotto, Amazarray, Chinazzo, \& Taborda, 2011; Gonçalves \& Kapcinski, 2008; Ludermir \& Lewis, 2005; Marín-León et al., 2007), nos quais os fatores sociodemográficos associados aos TMC têm evidenciado diferenças importantes entre homens e mulheres (Carlotto et al., 2011). No entanto a relação entre TMC e as variáveis laborais e estressores ocupacionais, ainda, é pouco explorada.

Ao discorrer sobre as diferenças da prevalência de transtornos mentais decorrentes do trabalho entre homens e mulheres, autores apontam para a baixa autoestima feminina e para o limitado controle das mulheres sobre os seus contextos de vida (Jenkins, 1985; Rosenfield, 1989). Discutem, ainda, a menor valorização do trabalho feminino na sociedade, fator que adiciona à prática laboral das mulheres um estresse adicional (Jenkins, 1985; Schraiber et al., 2005). Estudo realizado por Areias, Guimarães e Grubits (2000), com trabalhadores de uma universidade, verificou que os do sexo feminino apresentaram mais estressores externos do que os do sexo masculino, o que indica que as mulheres estão mais expostas aos efeitos danosos do estresse.

O adoecimento mental caracteriza-se por uma determinação complexa que envolve as dimensões econômica, social, política e cultural, expressando-se diferentemente em homens e mulheres (Lurdemir, 2008). Na abordagem dos problemas de saúde, deve-se, portanto, introduzir a perspectiva de gênero, considerando as diferenças no processo de socialização entre homens e mulheres, as quais determinam valores, atitudes e condutas diferenciadas (Borrell \& Artazcoz, 2008). Na maior parte dos estudos em saúde, a questão do gênero não tem sido incluída e quando considerada é utilizada para controle de seu efeito (Areias \& Guimarães, 2004). As autoras revelam que diversos estudos têm sido realizados com amostras de mulheres em ocupações de baixa qualificação, produzindo resultados com vieses que não explicam os efeitos do trabalho na saúde mental segundo o gênero feminino, em geral. A investigação desse impacto, portanto, deve ser conduzida através de pesquisas que considerem também a experiência de mulheres em ocupações mais qualificadas e valorizadas. 
O que essas pesquisas visam evidenciar é a interseccionalidade entre gênero e certos determinantes sociais da saúde, como classe, raça e escolaridade, entre outros. Desse modo, gênero se entrelaça a outros determinantes na produção da saúde ou doença decorrente dos processos laborais. Como outros determinantes é possível citar a valorização de determinadas ocupações em relação a outras, o papel ocupado pelo trabalho na vida de mulheres de estratos sociais distintos, bem como a precarização de determinadas formas de trabalho na atualidade (Araújo, Godinho, Reis \& Almeida, 2006). Tal perspectiva evidencia, portanto, que experiências laborais distintas resultam em maior ou menor adoecimento das mulheres.

Segundo Rohlfs (1999), os papéis atribuídos e assumidos por homens e mulheres na sociedade são importantes na explicação e compreensão de seu estado de saúde. Qualquer interpretação dos processos de trabalho e da forma de atuação laboral de homens e mulheres deve contemplar uma discussão acerca das relações de poder hegemônicas na sociedade, em que o trabalho feminino e o trabalho masculino são dotados de conotações e valorizações distintas.

As diferenças identificadas entre as formas de inserção no trabalho de homens e mulheres são complexificadas quando versamos sobre a natureza do trabalho desenvolvido, bem como das instituições onde esses trabalhadores desempenham suas funções laborais. Nesse sentido, estudos sobre a atividade de trabalho em uma instituição pública acrescenta à análise de gênero proposta uma necessária compreensão sobre as características de tais instituições.

\subsection{Trabalho e setor público}

As organizações públicas no Brasil, visando alcançar um desempenho mais produtivo, passaram, nos últimos anos, por um processo de profissionalização com mudanças substanciais em seu contexto e dinâmica de trabalho, decorrente principalmente da implantação de novas tecnologias e práticas gerenciais (Balassiano, Tavares, \& Pimenta, 2011). Embora o atual modelo de gestão se assemelhe aos adotados pela iniciativa privada, o setor público possui algumas especificidades quando comparado ao setor privado (Paiva \& Couto, 2008), pois tem objetivos, normas, produtos, usuários e recursos de origem diferenciados (Tohá \& Solari, 1997). As empresas públicas operam de forma sistemática, legal e técnica dos serviços característicos do Estado, em benefício da coletividade (Bem, Prado \& Delfino, 2013). Segundo a OECD (2004), essas diferenciam-se das empresas privadas por terem menor pressão competitiva, possuírem menos restrições orçamentárias, diferentes padrões de transparência e prestação de contas e forte influência política nas suas decisões estratégicas. Pires e Macêdo (2006) complementam referindo 
características como o burocratismo, o autoritarismo centralizado, o paternalismo, a falta de empreendedorismo, e a descontinuidade da gestão. Tais características da cultura de organizações públicas influenciam os seus trabalhadores, na medida em que reproduzem este funcionamento nas situações laborais e de organização do trabalho em comportamentos de apego às regras e rotinas, supervalorização da hierarquia, paternalismo nas relações, apego ao poder, dentre outras.

O profissional do setor público, apesar de possuir uma relação de trabalho menos instável, de menor exposição ao risco de demissão, está sujeito a outras formas de instabilidade e precarização do trabalho, tais como a privatização de empresas públicas, seguidas de demissão; a terceirização de setores dentro da empresa; a deterioração das condições de trabalho e da imagem do trabalhador do serviço público; e a responsabilização pelas deficiências dos serviços e por possíveis crises das instituições públicas. Está, ainda, exposto às instabilidades geradas por oscilações políticas e de planejamento, que ocasionam descontinuidade de ações, alterações na qualidade e quantidade da demanda pelos serviços ofertados, acúmulo de funções, mudanças na organização do trabalho ou na natureza das ações que conflitam com o sentido e as crenças que os trabalhadores têm em relação ao trabalho (Lancman, Sznelwar, Uchida \& Tuacek, 2007).

No campo da administração pública, no Brasil, ainda há a caréncia de pesquisas sobre o estresse ocupacional (Balassiano et al., 2011) e de sua associação com TMC (Macedo et al., 2007). Os autores afirmam que os efeitos do estresse ocupacional sobre a saúde do trabalhador em um ambiente em constante e intensa modificação ainda são pouco conhecidos e devem fazer parte da agenda de saúde pública. A produção científica no campo do trabalho e gênero ainda se mostra restrita e seu desenvolvimento é inicial, particularmente no tocante à saúde, assim, constituindo-se campo aberto para futuras explorações (Araújo et al., 2006).

Pelo exposto, o presente estudo pretendeu buscar a prevalência e evidências empíricas relativas à relação entre TMC, variáveis sociodemográficas e percepção de estressores ocupacionais em trabalhadores do setor público, assim, verificando se existe diferença entre homens e mulheres. Os resultados podem subsidiar novos estudos e ações específicas, para homens e mulheres, voltadas para a saúde e qualidade de vida do trabalhador, redução do absenteísmo e melhoria da qualidade dos serviços prestados.

\section{Método}

\subsection{Participantes}


A população em estudo constituiu-se de 8.185 servidores pertencentes ao quadro funcional do Poder Judiciário, em 2011, que desenvolviam seu trabalho em comarcas distribuídas em 164 cidades do estado do Rio Grande do Sul, incluindo a capital. A populaçãoalvo, calculada para um intervalo de confiança de $95 \%$, margem de erro de 5\%, assumindo-se como prevalência de TMC de 50\%, valor estimado quando não há informação sobre a proporção de interesse (Agranonik \& Hirakata, 2011) e 10\% para possíveis perdas, resultou em 679 sujeitos. A taxa de resposta foi $80,7 \%$, ou seja, 548 servidores. A amostra final constituiu-se de 541 participantes, pois foram excluídos 7 participantes que não responderam à questão relacionada ao sexo. Como critério de inclusão, considerou-se estar em atividade há mais de um ano.

A amostra constituiu-se de 354 mulheres $(65,4 \%)$ e 187 homens $(34,6 \%)$. Os servidores do sexo masculino, em sua maioria, não têm filhos $(63,7 \%)$, possuem companheira fixa $(79 \%)$ e formação em nível superior (77,2\%). A idade média desses profissionais é de 42 anos (DP = 9). Possuem, em média, 13 anos de trabalho na instituição ( $D P=9$ ). Quanto ao grupo do sexo feminino, 63,1\% não possuem filhos, $67,64 \%$ possuem companheiro fixo e formação em nível superior $(82,6 \%)$. As participantes têm, em média, 44 anos $(\mathrm{DP}=8)$ e apresentam média de 14 anos de trabalho na instituição $(\mathrm{DP}=8)$.

\subsection{Instrumentos}

Os dados foram coletados por meio dos seguintes instrumentos: (1) Questionário para levantamento de dados sociodemográficos (sexo, idade, escolaridade, situação conjugal) e laborais (tempo de trabalho, localidade) e percepção de estressores ocupacionais (carga horária, horário de trabalho, tipo de trabalho, diversidade de trabalho, complexidade do trabalho, relação com colegas, relação com chefia, condições físicas de trabalho, ambiente social, organização do trabalho). Os itens foram selecionados com base na literatura sobre o tema (Cooper, Sloan, \& Williams, 1988; Paschoal \& Tamayo, 2004) e validados por profissionais da instituição (assessores administrativos e diretor do departamento e médico da instituição da instituição). Os estressores foram avaliados por meio de uma escala de quatro pontos variando de 1 (não estressante) a 4 (muito estressante); (2) Self-Reporting Questionnaire (SRQ-20), instrumento que avalia ocorrência de TMC, desenvolvido por Harding et al. (1980) e validado, no Brasil, por Mari e Willians (1986). O instrumento é composto por 20 itens sobre sintomas físicos e psíquicos, com escala de respostas dicotômicas (sim/não) para detecção de distúrbios psiquiátricos menores (ansiedade, depressão, reações, 
psicossomáticas, irritação e cansaço mental). Cada resposta afirmativa pontua com o valor 1 para compor o escore final por meio do somatório desses valores. Os escores obtidos estão relacionados com a probabilidade de presença de TMC, variando de 0 (nenhuma probabilidade) a 20 (extrema probabilidade). O instrumento possui coeficiente alfa de Cronbach de 0,86 (Gonçalves et al., 2008), alta sensibilidade $(83 \%)$ e especificidade $(80 \%)$. O SRQ destina-se à detecção de sintomas e sugere apenas o nível de suspeição (presença/ausencia) de algum transtorno mental, mas não discrimina um diagnóstico específico (Santos, Araújo, \& Oliveira, 2009).

\subsection{Procedimentos}

A coleta de dados foi realizada por meio de uma avaliação on-line disponibilizada pela intranet da instituição em estudo, após aprovação do estudo pelo Comitê de Ética em Pesquisa da Pontifícia Universidade Católica do Rio Grande do Sul com número de protocolo $11 / 05483$. Os trabalhadores sorteados receberam um código/senha individual para acessar o sistema no período de uma semana.

O banco de dados foi digitado e analisado com o pacote estatístico Statistical Package for the Social Sciences (SSPS) versão 17.0. Foram realizadas análises descritivas de caráter exploratório para avaliar a qualidade do banco de dados, verificando possíveis erros de digitação, distribuição de itens, casos omissos e extremos. A análise dos dados para a prevalência de TMC foi realizada através do escore dicotômico do SRQ-20, foi adotado, neste estudo, o ponto de corte de 8, independente do sexo, utilizado por Gonçalves et al. (2008). Para a verificação de associação entre as variáveis sociodemográficas foi realizado o teste Qui-Quadrado (nível de significância de 5\%). A associação entre TMC e estressores ocupacionais foi avaliada por meio da prova $t$ de student. O tamanho do efeito foi calculado pela diferença média padronizada entre os grupos ( $d$ de Cohen), que considera 0,2 um valor indicativo de um efeito pequeno, $0,5 \mathrm{um}$ efeito médio e $0,8 \mathrm{um}$ tamanho de efeito grande (Cohen, 1992).

\section{Resultados}

Os grupos de homens e mulheres apresentaram diferença significativa nas variáveis situação conjugal e idade e não se diferenciaram nas demais características sociodemográficas (Tabela 1). 
Ta bela 1 - Comparação das caracteristicas sociodemográficas e la borais dos grupos de trabathadores

\begin{tabular}{|c|c|c|c|}
\hline \multirow[t]{2}{*}{ Variáveis } & Homens ( $\mathrm{n}=187$ ) & \multicolumn{2}{|c|}{ Mulheres $(n=354)$} \\
\hline & n $\%$ & $\%$ & $p$ \\
\hline \multicolumn{4}{|l|}{ Situação conjugal } \\
\hline Com companheiro & $113(79)$ & $205(67,4)$ & $0,001^{-}$ \\
\hline Sem companheiro & $30(21)$ & $99(32,6)$ & \\
\hline \multicolumn{4}{|l|}{ Idade } \\
\hline 21 a 40 anos & $83(44,6)$ & $110(31,7)$ & $0,001^{* *}$ \\
\hline 41 a 62 anos & $103(55,4)$ & $237(68,3)$ & \\
\hline \multicolumn{4}{|l|}{ Filhos } \\
\hline Nào & $114(63,7)$ & $207(63,1)$ & 0,448 \\
\hline Sim & $65(36,3)$ & $121(36,9)$ & \\
\hline \multicolumn{4}{|l|}{ Escolaridade } \\
\hline Ensino Medio & $42(22,8)$ & $60(17,4)$ & 0,085 \\
\hline Ensino Superior & $142(77,2)$ & $284(82,6)$ & \\
\hline \multicolumn{4}{|l|}{ Tempo de trabalho } \\
\hline Até 10 anos & $89(47,8)$ & $137(39,1)$ & 0,129 \\
\hline Entre 11 e 20 anos & $54(29,0)$ & $126(36,0)$ & \\
\hline Mais de 20 anos & $43(23,1)$ & $87(24,9)$ & \\
\hline \multicolumn{4}{|l|}{ Localidade } \\
\hline Capital & $99(52,9)$ & $189(53,5)$ & 0,990 \\
\hline Região Metropolitana & $18(9,6)$ & $34(9,6)$ & \\
\hline Interior & $70(37,4)$ & $130(36,8)$ & \\
\hline
\end{tabular}

$* * 0<0,01$

No que diz respeito à prevalência de TMC na população estudada (Tabela 2), os resultados evidenciaram maior percentual de mulheres com TMC $(n=164 ; 46,3 \%)$, sendo esta diferença estatisticamente significativa.

Tabela 2-Relação entre TMC e a variável sexo

\begin{tabular}{|c|c|c|c|c|c|c|}
\hline \multirow{2}{*}{ Variável } & \multirow{2}{*}{ Categoria } & \multicolumn{2}{|c|}{$\begin{array}{l}\text { Sem } \\
\text { TMC }\end{array}$} & \multicolumn{2}{|c|}{$\begin{array}{l}\text { Com } \\
\text { TMC }\end{array}$} & \multirow[t]{2}{*}{$p$} \\
\hline & & $\mathrm{n}$ & $\%$ & $\mathrm{n}$ & $\%$ & \\
\hline \multirow[t]{2}{*}{ Sexo } & Mulheres & 190 & 53,7 & 164 & 46,3 & $0,001 *$ \\
\hline & Homens & 128 & 68,4 & 59 & 31,6 & \\
\hline
\end{tabular}

$4 p<0,01$

Quanto à associação entre TMC e variáveis sociodemográficas de acordo com o sexo, não foram identificadas associações significativas (Tabela 3). 
Tabela 3 - Relação entre TMC e variá veis sociodemograficas de acordo com o sexo

\begin{tabular}{|c|c|c|c|c|c|c|c|c|c|c|c|}
\hline \multirow[t]{2}{*}{ Variável } & \multirow[t]{2}{*}{ Categoria } & \multicolumn{2}{|c|}{ Sem TMC } & \multicolumn{2}{|c|}{ Com TMC } & & \multicolumn{2}{|c|}{ Sem TMC } & \multicolumn{2}{|c|}{ Com TMC } & \\
\hline & & $\mathrm{n}$ & & $\mathrm{n}$ & $\%$ & & $\mathrm{n}$ & $\%$ & $\mathrm{n}$ & $\%$ & \\
\hline & & \multicolumn{5}{|c|}{ Matheres } & \multicolumn{4}{|c|}{ Homens } & \\
\hline \multirow[t]{2}{*}{ Situaçào conjugal } & Com companheiro & 111 & 54,1 & 94 & 45,6 & 0,443 & 80 & 70,8 & 33 & 29,2 & 0,785 \\
\hline & Sem companheiro & 52 & 52,5 & 47 & 47,5 & & 22 & 73,3 & 8 & 26,7 & \\
\hline \multirow[t]{2}{*}{ Idade } & 21 a 40 anos & 55 & 50,0 & 55 & 50,0 & 0,359 & 61 & 73,5 & 22 & 26,5 & 0,216 \\
\hline & 41 a 62 anos & 131 & 55,3 & 106 & 44,7 & & 67 & 65,0 & 36 & 35,0 & \\
\hline \multirow[t]{2}{*}{ Fithos } & $\operatorname{Sim}$ & 71 & 58,7 & 50 & 41,3 & 0,331 & 44 & 67,7 & 21 & 32,3 & 0,824 \\
\hline & Nāo & 110 & 53,1 & 97 & 46,9 & & 79 & 69,3 & 35 & 30,7 & \\
\hline \multirow[t]{2}{*}{ Escolaridade } & Médio & 34 & 56,7 & 26 & 43,3 & 0,489 & 28 & 66,7 & 14 & 33,3 & 0,841 \\
\hline & Superior & 147 & 51,8 & 137 & 48,2 & & 97 & 68,3 & 45 & 31,7 & \\
\hline
\end{tabular}

A análise da relação entre TMC e estressores ocupacionais revelou diferenças nas associações entre os grupos. A carga horária associouse aos TMC somente em homens e a diversidade e complexidade e o ambiente social do trabalho, somente em mulheres. Comum aos dois grupos foi perceber a chefia e os colegas como estressantes.

Tabela 4 - Relaçào entre TMC e estressores ocupacionais de acordo com o seso

\begin{tabular}{|c|c|c|c|c|c|c|c|c|c|}
\hline \multirow[t]{2}{*}{ Variaveis } & \multirow[b]{2}{*}{ TMC } & \multicolumn{4}{|c|}{ Homens ( $\mathrm{Sim}=123, \mathrm{Naz}=53)$} & \multicolumn{4}{|c|}{ Nilheres $(\operatorname{Sim}=162$, Nä0 $=187)$} \\
\hline & & $\mathrm{M}$ & DP & $p$ & $d$ & $M$ & DP & $p$ & $d$ \\
\hline \multirow[t]{2}{*}{ Carga Hotária } & $\mathrm{Nä0}$ & 1,13 & 0,50 & $0,003^{*}$ & 0.438 & 1,17 & 0,56 & 0,658 & 0,052 \\
\hline & Sim & 1,43 & 0,83 & & & 1,20 & 0,60 & & \\
\hline \multirow[t]{2}{*}{ Horärio } & Näo & 1,28 & 0,69 & 0,202 & 0.197 & 1,39 & 0,79 & 0,183 & 0,144 \\
\hline & Sim & 1,43 & 0,83 & & & 1,51 & 0,87 & & \\
\hline \multirow[t]{2}{*}{ Atendimento ao público } & Nào & 1,45 & 0,84 & 0,102 & 0.255 & 1,53 & 0,88 & 0,562 & 0,056 \\
\hline & $\operatorname{sim}$ & 1,68 & 0,96 & & & 1,58 & 0,91 & & \\
\hline \multirow[t]{2}{*}{ Diversidade } & Nào & 1,73 & 0,97 & 0,091 & 0.273 & 1,49 & 0,86 & $0,022^{*}$ & 0,336 \\
\hline & $\operatorname{Sim}$ & 2,00 & 1,01 & & & 1,80 & 0,98 & & \\
\hline \multirow[t]{2}{*}{ Complexidade do trabatho } & $\mathrm{Nà}$ & 1,70 & 0,96 & 0,121 & 0.254 & 1,42 & 0,81 & $0,001^{*}$ & 0,453 \\
\hline & Sim & 1,95 & 1,01 & & & 1,83 & 0,99 & & \\
\hline \multirow[t]{2}{*}{ Ambiente social } & Nào & 1,94 & 1,00 & 0,057 & 0.313 & 2,05 & 1,00 & $0,013^{*}$ & 0,277 \\
\hline & $\operatorname{Sim}$ & 2,25 & 0,98 & & & 2,32 & 0,95 & & \\
\hline \multirow[t]{2}{*}{ Colegas } & Näo & 1,46 & 0,84 & $0,007^{* *}$ & 0.433 & 1,45 & 0,84 & $0,001^{* *}$ & 0,425 \\
\hline & Sim & 1,86 & 1,00 & & & 1,84 & 0,99 & & \\
\hline \multirow[t]{2}{*}{ Chefias } & Näo & 2,27 & 0,97 & $0,044^{\circ}$ & 0.332 & 2,47 & 0,89 & $0,012^{\circ}$ & 0.270 \\
\hline & $\operatorname{Sim}$ & 2,57 & 0,83 & & & 2,69 & 0,73 & & \\
\hline \multirow[t]{2}{*}{ Condiçöes fisicas } & Năo & 1,11 & 0,47 & 0,425 & 0.133 & 1,01 & 0,15 & 0,245 & 0.137 \\
\hline & Sim & 1,18 & 0,58 & & & 1,04 & 0,27 & & \\
\hline
\end{tabular}

$* p<0,05 * p<0,01$ 


\section{Discussão}

O estudo buscou identificar a prevalência de TMC, bem como a relação entre TMC, variáveis sociodemográficas e a percepção de estressores ocupacionais, verificando se existe diferença entre homens e mulheres em servidores públicos de uma instituição do Poder Judiciário do estado do Rio Grande do Sul. Os resultados obtidos são consistentes com estudos prévios, nos quais a prevalência de TMC foi mais alta entre as mulheres (Bhui et al., 2001; Carlotto et al., 2011; Costa et al., 2002; Lima, Soares, \& Mari, 1999; Ludermir \& Lewis, 2005).

Conquanto variáveis sociodemográficas não tenham apresentado associação com o desenvolvimento de TMC na amostra investigada, verificaram-se diferenças nas características dessa amostra. Sobre a situação conjugal, os homens relataram estar mais envolvidos em relações estáveis do que as mulheres. Esse fato pode, em parte, representar para eles um fator protetivo ao desenvolvimento de TMC, uma vez que eles têm frequentemente com quem compartilhar as dificuldades cotidianas, inclusive aquelas advindas do ambiente do trabalho.

O suporte social encontrado dentro e fora do trabalho tem sido constantemente associado ao estresse ocupacional. Estudos revelam que a presença de redes sociais que oferecem suporte ao trabalho melhora a avaliação do empregado sobre a sua saúde e correlacionase negativamente com o estresse (Iwata \& Suzuki, 1997; Stansfeld, Shipley, Head, Fuhrer, \& Kivimaki, 2013).

Em contraste, $\mathrm{o}$ fato de as mulheres terem relatado menor envolvimento em relações afetivas estáveis aponta para uma possível sobrecarga dessas, resultante da necessária conciliação entre o trabalho público e o trabalho doméstico. Araújo, Pinho e Almeida (2005) afirmam que a inserção feminina no mercado formal de trabalho tem sido historicamente limitada pelas responsabilidades domésticas e familiares. Segundo Joshi (1990), ao contrário do que sucede com os homens, é o trabalho feminino que deve ser adaptado às funções de cuidado com a casa e os filhos, preocupações socialmente designadas às mulheres. Neste sentido, no que se refere à amostra do presente estudo, pode-se conjecturar que a sobrecarga advinda da conciliação do trabalho doméstico e produtivo seja vivenciada com especial intensidade por essas mulheres que não têm um companheiro para compartilhá-la.

Quando se compara a prevalência de TMC entre mulheres de diferentes estratos sociais e que, portanto, desempenham tarefas laborais diferentemente valorizadas, percebe-se uma relevante contradição. Embora o trabalho remunerado realizado fora do espaço da casa proteja a mulher do isolamento social e dos efeitos da baixa valorização do trabalho doméstico, este mesmo trabalho resulta em 
um conflito entre diferentes demandas, levando as mulheres ao estresse, à fadiga e a sintomas psíquicos (Bartley et al., 1992). Embora os homens e as mulheres que constituem a presente amostra desempenhem as mesmas atividades laborais, os resultados demonstraram diferenças entre os grupos no que se refere à relação entre TMC e estressores ocupacionais. Os homens perceberam a carga horária como um estressor, enquanto somente as mulheres perceberam o ambiente, a diversidade e a complexidade do trabalho como estressante. $O$ que os resultados sugerem é que o significado atribuído ao trabalho - e consequentemente aos seus estressores - é condicionado pela divisão sexual do trabalho (Abramo, 2007). Isto significa dizer que homens e mulheres percebem o trabalho de forma distinta, especialmente porque à atividade laboral masculina e feminina são atreladas expectativas diferentes.

Portanto é possível supor que a carga horária destinada ao trabalho produtivo seja sentida de forma particularmente intensa pelos homens, uma vez que tradicionalmente eles não dividem com as mulheres as preocupações com o trabalho reprodutivo. Segundo Bandeira et al. (2009), os homens destinam um número de horas bem inferior às mulheres aos afazeres domésticos não remunerados. Nesse sentido, o trabalho formal ocupa, para os homens, uma centralidade específica em suas vidas, expressa não somente pelo maior envolvimento com as tarefas laborais, mas também pela maior expectativa das chefias em relação a esse envolvimento. De modo oposto, o engajamento mais frequente das mulheres com o trabalho doméstico, possivelmente, faça com que elas cumpram estritamente a sua jornada laboral, dispensando o restante de seu tempo e energia aos cuidados com a casa e a prole.

Sobre o ambiente social do trabalho e sua associação com o desenvolvimento de TMC em mulheres, a literatura de gênero aponta as mulheres como seres primordialmente relacionais (Miller, 1986; Gilligan, 1982). Neste sentido, o processo de construção subjetiva das mulheres é permeado por valores como a ênfase no relacionamento interpessoal, o foco no afeto, na intimidade e na formação de vínculos. Para Oliveira (1993), a valorização da empatia, da sensibilidade e da interação com os outros faz com que as mulheres encontrem dificuldades no acesso ao espaço público. Nesta ocasião, segundo a autora, "as mulheres se vêem obrigadas a confrontar seu modo de ser com a exigência de sucesso no mundo dos homens, marcado por agressividade, competitividade, objetividade e eficiência" (p. 47).

No mundo do trabalho, tais características socialmente atribuídas às mulheres - como empatia, sensibilidade, afetividade e cuidado com o outro - expressam-se usualmente na maior preocupação feminina com a qualidade do ambiente de trabalho. Lombardi (2008), sobre o estilo feminino de inserção no mundo masculino do trabalho, destaca 
que as mulheres comumente se preocupam em tornar o ambiente de trabalho mais amigável, menos tenso e mais caloroso.

Se, por um lado, a inserção das mulheres no mundo masculino do trabalho representa a possibilidade de humanização dos ambientes laborais (Lombardi, 2008), por outro, a expectativa de que as mulheres criem espaços de trabalho especialmente agregadores $\mathrm{e}$ calorosos reforça os estereótipos de gênero que posicionam as mulheres como não agressivas e não competitivas. Assim, um ambiente hostil de trabalho, pode-se conjecturar, apresenta-se como potencialmente adoecedor para as mulheres no sentido de que representa uma ameaça à própria subjetividade feminina, socialmente associada à docilidade e ao afeto no trato com aqueles ao seu redor.

No que se refere às características do trabalho desenvolvido, as mulheres da amostra indicaram a diversidade e a complexidade das tarefas desenvolvidas como estressores no trabalho. Tal resultado pode ser uma vez mais discutido sob a perspectiva dos estereótipos masculinos e femininos e às formas como eles se expressam no mundo do trabalho. Homens são socialmente percebidos como mais racionais, assertivos e pragmáticos (Vianna, 2013). Tais características são comumente valorizadas no ambiente laboral, por, supostamente resultar em ações mais efetivas e lucrativas.

Embora a polivalência e a atenção aos detalhes, características associadas ao trabalho feminino, sejam valorizadas como atributos positivos, Carilho (2013) enfatiza que elas não resultam em maiores salários ou melhores condições de ascensão para as mulheres no ambiente de trabalho. Segundo a autora, tais características são consideradas como naturais às mulheres, não como atributos que devam ser especificamente valorizados no trabalhador.

No que diz respeito aos resultados do presente estudo, é possível que a complexidade e a diversidade de tarefas para as mulheres resultem em um sentimento de sobrecarga, uma vez que elas comumente tendem a deterem-se aos detalhes da consecução de tais tarefas. Em contraste, os homens, diante de demandas da mesma natureza, provavelmente, têm uma postura mais assertiva e resolutiva, o que resulta em ações menos onerosas do ponto de vista emocional.

Comum aos dois grupos foi perceber a chefia e colegas como estressantes. A relação com chefias e colegas tem sido apontada em diferentes estudos como um importante estressor ocupacional. Tal resultado contraria estudos que evidenciam que mulheres tendem a referir estressores interpessoais com maior frequência que homens (Mazzola, Schonfeld, \& Spector, 2011) e maior ocorrência de licenças médicas de longo prazo devido a problemas de saúde mental decorrentes de estressores psicossociais em mulheres (Glise, Ahlborg, \& Jonsdottir, 2012). Este resultado pode ser entendido a partir do perfil da amostra investigada, uma vez que os profissionais 
possuem características bastante similares em termos de formação profissional e tempo de trabalho. Também, pode-se pensar nas questões relacionadas à carreira do servidor público que oferece oportunidades similares a homens e mulheres.

\subsection{Forças, limitações do estudo e implicações para a prática}

Uma das forças do presente estudo é a utilização de um construto teórico consolidado e que possui um instrumento de avaliação adaptado e validado para o uso no Brasil. Ressalta-se o fato de que a amostra é probabilística, o que possibilita a generalização de seus resultados para a população em estudo.

O estudo apresenta limitações que devem ser consideradas para a leitura de seus resultados. Trata-se de um estudo transversal que impossibilita estabelecer causalidades. Foram utilizadas medidas autorreferidas que podem conduzir a um viés de resposta, de aumento ou de minimização. Embora os resultados possam ser generalizados para a população em estudo, esses são decorrentes de uma organização pública localizada em uma região específica da região Sul do Brasil, não sendo, portanto, passíveis de generalizações para outras instituições públicas. É importante considerar, ainda, o "efeito do trabalhador sadio", questão peculiar em estudos transversais em epidemiologia que, muitas vezes, exclui o possível doente (Mc Michael, 1976). Esta é uma situação que pode subestimar - tamanho dos riscos identificados porque os mais afetados não conseguem se manter no emprego, geralmente afastando-se por licenças para tratamento de saúde.

\section{Referências}

Agranonik, M., \& Hirakata, V. N. (2011). Cálculo de tamanho de amostra: proporções. Revista HCPA, 31(3), 382-388.

Abramo, L. W. (2007). A inserção da mulher no mercado de trabalho: uma força de trabalho secundária? Tese de doutorado, Universidade de São Paulo, São Paulo, SP, Brasil.

Apple, M. W. (1995). Trabalho docente e textos: economia política das relações de classe e de gênero em educação. Porto Alegre: Artes Médicas.

Araújo, T. M., Aquino, E., Menezes, G., Santos, C. O., \& Aguiar, L. (2003). Aspectos psicossociais do trabalho e distúrbios psíquicos entre trabalhadores de enfermagem. Revista de Saúde Pública, 37, 424-433.

Araújo. T. M., Pinho, P. S., \& Almeida, M. M. G. (2005). Prevalência de transtornos mentais comuns em mulheres e sua relação com 
as características sociodemográficas e o trabalho doméstico. Revista Brasileira de Saúde Materno Infantil, 5(3), 337-348.

Araújo, T. M. de, Godinho, T. M., Reis, E. J. F. B. dos, \& Almeida, M. M. G, de. (2006). Diferenciais de gênero no trabalho docente e repercussões sobre a saúde. Ciência \& Saúde Coletiva, 11(4), 1117-1129.

Areias, M. E. Q., Guimarães, L. A. M., \& Grubits, S. (2000). Stress and support in workers at a Brazilian university. In Books of abstracts. XXVII International Congress of Psychology (p. 78). Stockholm.

Areias, M. E. Q., \& Guimarães, L. A. M. (2004). Gênero e estresse em trabalhadores de uma universidade pública do estado de São Paulo. Psicologia em Estudo, 9(2), 255-262.

Balassiano, M., Tavares, E., \& Pimenta, R. da C. (2011). Estresse ocupacional na administração pública brasileira: quais os fatores impactantes? Revista de Administração Pública, 45(3), 751-74.

Bandeira, L., Melo, H. P., \& Pinheiro, L. S. (2009). Mulheres em dados: o que informa a Pnad/IBGE, 2008. In: BRASIL, Presidência da República. Edição Especial Revista do Observatório Brasil da Igualdade de Gênero: Autonomia Econômica, Empoderamento e Inserção das Mulheres no Mercado de Trabalho. Brasília/DF: SPM, p.107-119. Recuperado em 10 de dezembro, 2014, de http://www.observatoriodegenero.gov.br/menu/areastematicas/trabalho/trabalho-1

Barnett, R. C., Marshall, N. L., Raudenbush, S. W., \& Brennan, R. T. (1993). Gender and the relationship between job experiences and psychological distress: a study of dual-earner couples. Journal of Personality and Social Psychology, 64(5), 794-806.

Bartley, M., Popay, J., \& Plewis, I. (1992). Domestic conditions, paid employment and women's experience of ill-health. Sociology of Health and I/Iness, 14, 313-343.

Bem, R. M. Prado, M. L., \& Delfino, N. (2013). Desafios à implantação da gestão do conhecimento: a questão cultural nas organizações públicas federais brasileiras. Revista Digital de Biblioteconomia e Ciência da Informação, 11(2), 123-135.

Bhui, K., Stansfeld, S. McKenzie, K., Karlsen, S., Nazroo, J., \& Scott Weich, S (2001). Racial/ethnic discrimination and common mental disorders among workers: findings from the empiric study of ethnic minority groups in the United Kingdom. American Journal of Public Health, 95(3), 496-501.

Borrell, C., \& Artazcoz, L. (2008). Las desigualdades de género en salud: retos para el futuro. Revista Española de Salud Pública, 82(3), 241-249. 
Borsoi, I. C. F. (1995). A saúde da mulher trabalhadora. In W. Codo \& J. J. C. Sampaio. (Orgs.), Sofrimento psíquico nas organizações - Saúde Mental e Trabalho (pp. 115-126). Petrópolis: Vozes.

Broadhead, W. E., Blazer, D. G., George, L. K., \& Tse, C. K. (1990). Depression, disability and days lost from work. JAMA, 264(19), 2524-2528.

Carrilho, A. (2013). A feminização na produção científica recente: um conceito difuso de compreensão necessária. In S. C. Yannoulas (Org.), Trabalhadoras: análise da feminização das profissões e ocupações (pp. 281-298). Brasília: Abaré Editorial.

Carlotto, M. S., Amazarrav, M. R., Taborda, L., \& Chinazzo, I. R. (2011). Transtornos Mentais Comuns e fatores associados em trabalhadores: uma análise na perspectiva de gênero. Cadernos Saúde Coletiva (UFRJ), 19, 172-178.

Cohen, J. (1992). A power primer. Psychological Bulletin, 112, 155159.

Cooper, C. L., Sloan, S. J., \& Williams, S. (1988). Occupational stress indicator management guide. Windsor: NFER-Nelson.

Costa, J. S. D. de et al. (2002) Prevalência de distúrbios psiquiátricos menores na cidade de Pelotas. Revista Brasileira de Epidemiologia, 5(2), 164-173.

Cyrino, R. (2009). Trabalho, temporalidade e representações sociais de gênero: uma análise da articulação entre trabalho doméstico e assalariado. Sociologias, 21, 66-92.

Diogo, M. F., \& Coutinho, M. C. (2006). A dialética da inclusão/exclusão e o trabalho feminino. Interaç,ões, 11(21), 121-142.

Divya, R., Girija K., Tintu T., \& Santhosh M. (2013). Influence of occupational stress on health among the medical trancriptors. International Journal of Pharma \& Bio Sciences, 4(1), 11011106.

European Agency on Safety and Health at Work (2007). Expert Forecast on Emerging Psychosocial Risks Related to Occupational Safety and Health. Luxembourg: European Communities.

Fonseca, M. L. G., Guimarães, M. B. L., \& Vasconcelos, E. M. (2008). Sofrimento difuso e transtornos mentais comuns: uma revisão bibliográfica. Revista de Atenção Primária à Saúde, 11(3), 285294.

Gonçalves, D. M., \& Kapczinski, F. (2008). Mental disorders in a community assisted by the Family Health Program. Cadernos de Saúde Pública, 24(7), 1641-1650.

Goldberg, D., \& Huxley, P. (1992). Common mental disorders: a biosocial model. New York: Tavistock/Routledge. 
Glise, K., Ahlborg, G., \& Jonsdottir, I. H. (2012). Course of mental symptoms in patients with stress-related exhaustion: does sex or age make a difference? BMC Psychiatry, 12(18). Recuperado em 25 de junho, 2014, de http://www.biomedcentral.com/content/pdf/1471-244X-1218.pdf

Gilligan, C. (1982). In a different voice: psychological theory and women's development. Cambridge: Harvard University Press.

Harding, T. W. et al. (1980). Mental disorders in primary health care: a study of their frequency and diagnosis in four development countries. Psychological Medicine, 10, 231-241.

Heilborn, M. L (2004). Dois é par: gênero e identidade sexual em contexto igualitário. Rio de Janeiro: Editora Garamond.

Iwata, N., \& Suzuki, K. (1997). Role stress-mental health relations in Japanese bank workers: a moderating effect of social support. Applied Psychology: An International Review, 46, 207-218.

Jenkins, R. (1985). Sex difference in minor psychiatric morbidity. Cambridge: Cambridge University Press.

Joshi, H. E. (1990). Changing roles of women in the British labour market and the family. In P. Deane (Org.), Frontiers of Economic Research (pp. 101-128). London: Macmillan.

Koltermann, I. T. A. P., Tomasi, E., Horta, B. L., \& Koltermann, A. P. (2011). Estresse ocupacional em trabalhadores bancários: prevalência e fatores associados. Saúde (Santa Maria), 37(2), 33-48.

Lancman, S., Sznelwar, L. I., Uchida S., \& Tuacek, T. A. (2007) O trabalho na rua e a exposição à violência no trabalho: um estudo com agentes de trânsito. Interface, 1(2I), 79-92.

Leka, S., Griffiths, A., \& Cox, T. (2003). Work organisation and stress. Geneva: World Health Organization.

Lima, M. S., Soares, B. G. O., \& Mari, J. J. (1999). Saúde e doença mental em Pelotas, RS: dados de um estudo populacional. Revista de Psiquiatria Clínica, 26(5), 225-235.

Lima, M. S, Beria, J. U., Tomasi. E., Conceição, A.T., \& Mari, J.J. (1996). Stressful life events and minor psychiatric disorders: an estimate of the population attributable fraction in a Brazilian community-based study. International Journal of Psychiatry in Medicine, 26, 211-222.

Lombardi, M. R. (2008). Engenheira e gerente: desafios enfrentados por mulheres em posições de comando na área tecnológica. In A. Costa, B. Sorj, C. Bruschini, \& H. Hirata (Orgs.), Mercado de trabalho e gênero: comparações internacionais (pp. 387-402). Rio de Janeiro: FGV.

Lopes, C. S., Faerstein, E., \& Chor, D. (2003). Eventos de vida produtores de estresse e Transtornos Mentais Comuns: 
resultados do Estudo Pró-Saúde. Cadernos de Saúde Pública, 19(6), 1713-1720.

Ludermir, A. B., \& Melo, D. A. F. (2002). Condições de vida e estrutura ocupacional associadas a transtornos mentais comuns. Revista de Saúde Pública, 36(2), 213-221.

Ludermir, A. B., \& Lewis G. (2005). Is there a gender difference on the association between informal work and common mental disorders? Social Psychiatry and Psychiatric Epidemiology, $40(8), 622-627$.

Ludermir, A. B. (2008). Desigualdades de classe e gênero e saúde mental nas cidades. Physis, 18(3), 451-467.

Macedo, L. E. T., Chor, D., Andreozzi, V., Faerstein, E., Werneck, G. L., \& Lopes, C. S. (2007). Estresse no trabalho e interrupção de atividades habituais, por problemas de saúde, no Estudo PróSaúde. Caderno de Saúde Publica, 23(10), 2327-2336.

McMichael, A. J. (1976). Standardized mortality ratios and the healthy worker effect: scratching beneath the surface. Journal of Occupational Medicine, 18(3), 165-168.

Mari, J. J., \& Jorge, M. R. (2004) Transtornos psiquiátricos na clínica geral. Psychiatry on line. Recuperado em 15 de maio, 2013, de http://www.polbr.med.br/arquivo

Mari, J. J., \& Williams, P. (1986). Misclassification by psychiatric screening questionnaires. Journal of Chronic Diseases, 39(5), 371-377.

Marín-León, L. Oliveira, H.B. de, Barros, M. B. de A., Dalgalarrondo, P., \& Botega, N. J. (2007). Social inequality and common mental disorders. Revista Brasileira de Psiquiatria, 29(3), 250253.

Mazzola, J. J., Schonfeld, I. S., \& Spector, P. E. (2011). What qualitative research has taught us about occupational stress. Stress and Health, 27(2), 93-110.

Miller, J. B. (1986). Towards a new psychology of women. Boston: Beacon Press.

Ministério da Saúde. (2001). Portaria no 95 de 26 de Janeiro de 2001. Dispõe sobre a NOAS Norma Operacional da Assistência à Saúde. Brasília: MS.

Moreno, N. (1999). Condiciones de trabajo y salud desde uma perspectiva de gênero. I Jornada de la Red de Médicas y Profesionales de la Salud, Madrid, 9, 64-70.

Narvaz, M. G., \& Koller, S. H. (2006). Famílias e patriarcado: da prescrição normativa à subversão criativa. Psicologia $e$ Sociedade, 18(1), 49-55.

Nieuwenhuijsen, K., Bruinvels, D., \& Frings-Dresen, M. (2010). Psychosocial work environment and stress-related disorders, a systematic review. Occupational Medicine, 60, 277-286. 
Organisation for Economic Co-operation and Development [OECD] (2004). Principles of corporate governance. Recuperado em 29 de maio de 2015 de http://www.oecd.org/daf/ca/corporategovernanceprinciples/315 57724.pdf

Oliveira, R. D. (1993). Elogio da diferença. O feminino emergente. São Paulo: Brasiliense.

Paschoal, T., \& Tamayo, A. (2004). Validação da escala de stress no trabalho. Estudos de Psicologia, 9(1), 45-52.

Paiva, K. C. M. De, \& Couto, J. H. (2008). Qualidade de vida e estresse gerencial "pós-choque de gestão": o caso da CopasaMG. Revista de Administração Pública, 42(6), 1189-1211.

Pires, J. C. S., \& Macedo, K. B. (2006). Cultura organizacional em organizações públicas no Brasil. Revista Brasileira de Administração Pública, 40(1), 81-105.

Porto, L. A., Carvalho, F. M., Oliveira, N. F. de, Silvany Neto, A. M., Araújo, T. M. de, Reis, E. J. F. B. dos, \& Delcor, N. S.. (2006). Associação entre distúrbios psíquicos e aspectos psicossociais do trabalho de professores. Revista de Saúde Pública, 40(5), 818-826.

Rohlfs, D. (1999). La perspectiva de género en el estudio de las diferencias y desigualdades en salud. I Jornada de la Red de Médicas y Profesionales de la Salud, Madrid, 12-13.

Rosenfield, S. (1989). The effects of women's employment personal control and sex differences in mental health. Journal of Health and Social Behavior, 30, 77-91.

Santos, K. O. B., Araújo, T. M. de, \& Oliveira, N. F. de. (2009). Estrutura fatorial e consistência interna do Self-Reporting Questionnaire (SRQ-20) em população urbana. Cadernos de Saúde Pública, 25(1), 214-222.

Schraiber, L. B., d'Oliveira, A. F. P. L., Falcão, M. T. C., \& Figueiredo, W. S. (2005). Violência dói e não é direito: a violência contra a mulher, a saúde e os direitos humanos. São Paulo: Editora Unesp.

Stansfeld, S. A., Shipley, M. J., Head, J., Fuhrer, R., \& Kivimaki, M. (2013). Work characteristics and personal social support as determinants of subjective well-being. PLOS ONE 8(11), e81115. Recuperado em 27 de maio, 2015, de http://www.plosone.org/article/fetchObject.action?uri=info:doi/ 10.1371/journal. pone.0081115\&representation =PDF

Szeto, A. C. H., \& Dobson, K. S. (2013). Mental disorders and their association with perceived work stress: an investigation of the 2010 Canadian Community Health Survey. Journal of Occupational Health Psychology, 18(2), 191-197. 
Silva, A. T. C., \& Menezes, P. R. (2008). Esgotamento profissional e transtornos mentais comuns em agentes comunitários de saúde. Revista de Saúde Pública, 42, 921-929.

Tohá, C., \& Solari, R. (1997). La modernización del estado y la gerencia pública. Santiago, Chile: Fundación Friedrich Ebert.

Theorell, Hammarström, Gustafsson, Hanson, \& Westerlund (2014). Job strain and depressive symptoms in men and women: a prospective study of the working population in Sweden. Journal of Epidemiology \& Community Health, 68, 78-82.

Vianna, C. P. (2013). A feminização do magistério na educação básica e os desafios para a prática e a identidade coletiva docente. In S. C. Yannoulas (Org.). Trabalhadoras: análise da feminização das profissões e ocupações (pp. 159-180). Brasília: Abaré Editorial.

\section{Endereço para correspondência \\ Mary Sandra Carlotto}

Pontifícia Universidade Católica do Rio Grande do Sul

Programa de Pós-Graduação em Psicologia

Av. Ipiranga, 6681, Partenon, CEP 90619-900, Porto Alegre - RS, Brasil

Endereço eletrônico: mscarlotto@gmail.com

\section{Mariana Barcinski}

Pontifícia Universidade Católica do Rio Grande do Sul

Faculdade de Psicologia

Av. Ipiranga, 6681, Partenon, CEP 90619-900, Porto Alegre - RS, Brasil

Endereço eletrônico: mbarcinski@gmail.com

\section{Rosália Fonseca}

Tribunal de Justiça do Estado do Rio Grande do Sul

Av. Borges de Medeiros, 1565, CEP 90110-906, Porto Alegre - RS, Brasil

Endereço eletrônico: rosaliafonseca7@gmail.com

Recebido em: 09/03/2015

Reformulado em: 04/06/2015

Aceito para publicação em: 09/06/2015

\section{Notas}

* Psicóloga, mestre em Saúde Coletiva, doutora em Psicologia Social.

** Psicóloga, mestre em Psicossociologia de Comunidades e Ecologia Social, doutora em Developmental Psychology.

*** Psicóloga, especialista em Saúde do Trabalhador. 